\title{
Effects of combined radiosurgery and temozolomide therapy on epidermal growth factor receptor and variant III in glioblastoma multiforme
}

\author{
YIGUANG LIN $^{1 *}$, JUNHU ZHOU $^{2 *}$, JIANGLONG XU $^{3 *}$, KAI ZHAO $^{1}$, XIAOMIN LIU ${ }^{4}$, \\ GUOKAI WANG ${ }^{1}$, ZHIYUAN ZHANG $^{1}$, YOULIN GE ${ }^{1}$, YONGQING ZONG $^{1}$, DESHENG XU $^{1}$, \\ YANLI TAN $^{3}$, CHUAN FANG $^{3}$ and CHUNSHENG KANG ${ }^{2}$
}

\begin{abstract}
${ }^{1}$ Gamma Knife Centre, Department of Neurosurgery, The Second Hospital of Tianjin Medical University, Tianjin 300211;
${ }^{2}$ Tianjin Neurological Institute, Department of Neurosurgery, Tianjin Medical University,

General Hospital and Laboratory of Neurooncology, Tianjin 300052; ${ }^{3}$ Department of Neurosurgery,

Affiliated Hospital of Hebei University, Baoding, Hebei 071000; ${ }^{4}$ Gamma Knife Centre, Department of Neurosurgery,

Tianjin Huanhu Hospital, Tianjin 300350, P.R. China
\end{abstract}

Received March 11, 2016; Accepted January 19, 2018

DOI: $10.3892 / \mathrm{ol} .2018 .8055$

\begin{abstract}
Glioblastoma multiforme (GBM) is a highly malignant and notably aggressive primary tumour. Variant III of the epidermal growth factor receptor (EGFRvIII) is one of the most common types of variants in GBM, and serves an important role in tumour invasion, proliferation and treatment resistance. In the present study, statistical analyses were performed on data from 57 patients with GBM, and polymerase chain reaction detection was conducted on the tumour tissues from 32 of these patients. The results indicated that the EGFRvIII mutation was significantly associated with tumour malignancy. Human GBMU87-EGFRvIII cell lines were cultured and treated with radiosurgery and temozolomide individually, or with combined radiosurgery and temozolomide treatment. In vitro and in vivo experimental methods were used to detect the expression levels of Ki-67 and EGFRvIII. As verified in the present study, the EGFRvIII mutation is positively correlated with the malignancy of tumours, and combined radiosurgery and temozolomide therapy may inhibit the invasion and
\end{abstract}

Correspondence to: Professor Xiaomin Liu, Gamma Knife Centre, Department of Neurosurgery, Tianjin Huanhu Hospital, 6 Jizhao Road, Tianjin 300350, P.R. China

E-mail: liuxiaomintj@126.com

Professor Chuan Fang, Department of Neurosurgery, Affiliated Hospital of Hebei University, 212 Yuhua East Road, Baoding, Hebei 071000, P.R. China

E-mail: fangchuan103@126.com

${ }^{*}$ Contributed equally

Key words: epidermal growth factor receptor variant III, variant, radiation treatment, temozolomide, invasion, proliferation proliferation abilities of U87- EGFRvIII more effectively than treatment alone.

\section{Introduction}

With an incidence rate of 3.19/100,000, glioblastoma multiforme (GBM) is a highly malignant and notably aggressive primary tumour of the human brain, and the median survival time for patients is $<12$ months in the majority of cases. The invasion of tumour cells into normal brain tissues means that it is difficult for surgery to achieve complete resection, and recurrence and mortality often occur. Different genetic aberrations arising from GBM lead to genetic heterogeneity of patient tumour cells, which infers resistance to additional therapies, including post-operative radiotherapy and chemotherapy (1-3). Epidermal growth factor receptor variant III (EGFRvIII) is one of the most common types of mutations of GBM and is responsible for various biological effects on the generation and development of tumours. Previous studies have indicated that this variation is significantly associated with poor prognosis in patients with GBM. Previously, numerous studies have indicated that EGFRvIII is associated with multiple characteristics of glioma stem cells, including self-renewal, abnormal differentiation, tumour initiation and treatment resistance. Studies have also indicated that high expression levels of EGFRvIII are positively correlated with the invasion abilities of GBM cells $(1,4)$. The amplification of EGFR is often accompanied by upregulated expression levels of EGFRvIII, which is significantly correlated with a short survival time for patients with BMG $(5,6)$. EGFRvIII is also expressed in other types of cancer, including lung cancer, but has not been identified in normal tissues or benign tumour tissues (7). Those tumours exhibiting activated EGFRvIII account for $\sim 50 \%$ of patients with BMG $(8,9)$. These mutations are not detected in the normal brain tissues or in other normal tissues of adult humans $(10,11)$. Thus, due to its biological characteristics, EGFRvIII may serve as a good target for the gene therapy of GBM. If an antibody that specifically 
recognizes EGFR and induces apoptosis is designed, it may have the potential to effectively kill tumour cells through coupling with cytotoxic drugs. However, only limited studies exist on the molecular biology of EGFRvIII-associated mechanisms. Following continuous studies and the development of target drugs for clinical applications, the combination of surgery and chemoradiotherapy remains the main measure of current clinical therapies. Specifically, combined therapy using radiation treatment and temozolomide is the main regimen for GBM treatment. For GBM with the EGFRvIII mutation, the response to combined radiation treatment and temozolomide therapy remains unknown. To the best of our knowledge, there have been no studies investigating the mechanisms of the development of resistance. Therefore, the present study collected clinical materials and used a combination of in vitro and animal models to examine the effects of combined radiation treatment and temozolomide therapy on patients with GBM with the EGFRvIII mutation.

\section{Materials and methods}

Cell lines and tissue samples. A total of 57 glioma patients with complete follow-up results who were treated between January 2013 and February 2014 in the Affiliated Hospital of Hebei University (Baoding, China) were randomly selected (Table I). Amongst the patients, 30 were men and 27 were women, with ages ranging from 22 to 76 years and a median age of 49 years. The Karnofsky Performance Status (KPS) scores for these patients ranged from 90 to 20 points, with a mean of 73 points. A total of 34 patients underwent a complete resection under surgical microscopy and 23 patients underwent a partial resection. A total of 26 patients exhibited tumors growing in the left brain and 31 patients exhibited tumors growing in the right brain. Post-operative pathological results indicated that 21 tumors were World Health Organization (WHO) grade I-II, 22 were WHO grade III and 14 were WHO grade IV (11). Statistical analyses were conducted on the clinical materials, and the Affiliated Hospital of Hebei University provided 32 tumor specimens preserved in liquid nitrogen. Amongst the 32 patients selected, 13 were men and 19 were women, with ages ranging from 23 to 71 years, with a mean age of 47 years. The post-operative pathological results indicated that 10 patients were WHO grade II, 5 patients were WHO grade II-III, 8 patients were WHO grade III and 9 patients were WHO grade IV, and 7 exhibited positive EGFRvIII results. The human GBM U87-EGFRvIII cell lines were obtained from Dr Webster Cavenee (Ludwig Cancer Institute, San Diego, CA, USA). The cells were grown in Dulbecco's modified Eagle's medium (Gibco; Thermo Fisher Scientific, Inc., Waltham, MA, USA) supplemented with $10 \%$ fetal bovine serum (Invitrogen; Thermo Fisher Scientific, Inc.) and $0.4 \%$ Geneticin (G-418 sulphate; Gibco; Thermo Fisher Scientific, Inc.). The cells were incubated at $37^{\circ} \mathrm{C}$ in a $5 \% \mathrm{CO}_{2}$ and $95 \%$ air environment until use. Approval for the use of the glioma samples was obtained from each patient and the present study was approved by the Ethics Committee of the Second Hospital of Tianjin Medical University (Tianjin, China).

Cell Counting Kit-8 (CCK-8) assay. The cells were seeded in 96-well plates at a density of $5 \times 10^{3}$ cells/well, with each well containing $100 \mu \mathrm{l}$ cell suspension. Subsequent to cell attachment, temozolomide (Tasly Pharmaceutical Co., Ltd., Tianjin, China) was added at different concentration gradients ( 0.1 to 1 , at intervals of 0.1 and 1 to 10 , at intervals of 1). After $72 \mathrm{~h}$, $10 \mu$ l CCK-8 reagent (Dojindo Molecular Technologies, Inc., Kumamoto, Japan) was added to each well, and the plates were incubated at $37^{\circ} \mathrm{C}$ for $4 \mathrm{~h}$. The optical absorbance at $450 \mathrm{~nm}$ was measured on a microplate reader. The half maximal inhibitory concentration $\left(\mathrm{IC}_{50}\right)$ result for $72 \mathrm{~h}$ was calculated using Origin 7.5 software (Microcal Software, Northampton, MA, USA). The $\mathrm{IC}_{50}$ of the U87-EGFRvIII cell line following $72 \mathrm{~h}$ of exposure to temozolomide, as measured by the CCK-8 kit was $5 \mathrm{mmol} / 1$.

RNA extraction and polymerase chain reaction (RT-PCR). Total RNA from the patient GBM tissues was extracted using the TRIzol reagent (Invitrogen; Thermo Fisher Scientific, Inc.) according to the manufacturer's protocol. The concentration and purity of RNA were determined using a NanoDrop ${ }^{\circledR}$ ND-1000 spectrophotometer (Thermo Fisher Scientific, Inc.). The GoScript ${ }^{\mathrm{TM}}$ Reverse Transcriptase system (Promega Corporation, Madison, WI, USA) was used in complementary DNA synthesis according to the manufacturer's instructions. The expression levels of mature EGFRvIII were determined by semi-quantitative PCR using a PCR cycler (Nanjing KeyGenBiotech, Co., Ltd., Beijing, China) with a forward primer for the mature DNA sequence and a universal adaptor reverse primer as follows: Forward, 5'-TGACTCCGTCCA GTATTGATCG-3' and reverse, 5'-GCCCTTCGCACTTCT TACACTT-3' (Thermo Fisher Scientific Inc.). All PCR was performed using a PCR kit (PrimeSTAR ${ }^{\circledR}$ Max DNA Polymerase; cat. no. R045A; Takara Biotechnology Co., Ltd., Dalian, China), according to the manufacturer's instructions. The PCR cycling conditions were as follows: 1 cycle at $95^{\circ} \mathrm{C}$ for $10 \mathrm{~min} ; 35$ cycles at $95^{\circ} \mathrm{C}$ for $30 \mathrm{sec}, 58^{\circ} \mathrm{C}$ for $30 \mathrm{sec}$ and $72^{\circ} \mathrm{C}$ for $1 \mathrm{~min}$; and 1 cycle at $72^{\circ} \mathrm{C}$ for $10 \mathrm{~min}$. The melting curve was obtained from $72-95^{\circ} \mathrm{C}$. The data were derived from three independent experiments.

Agarose gel electrophoresis. A 0.5\% agarose gel was prepared, and poured onto a taped plate with casting combs in place. A total of 20-30 min was allowed for the gel to set. The tape and the gel casting combs were carefully removed, and the gel was placed in a horizontal electrophoresis apparatus. A 1XTris base, acetic acid and EDTA electrophoresis buffer was added to the reservoirs until the buffer covered the agarose gel. Loading dye (ethidium bromide) was added to each DNA sample, and the samples were mixed and placed into the wells. The gel was run at 150-200 mA until the required separation was achieved in $30 \mathrm{~min}$. The DNA fragments were visualized on a long-wave ultraviolet light box and images were captured with a camera.

Cell treatment. For the radiation treatment, collected cells were digested in a sterile 1.5-ml Eppendorf (EP) tube on a super clean bench and centrifuged for $5 \mathrm{~min}$ at $\sim 63 \mathrm{x} \mathrm{g}$ (room temperature) with a tablet centrifuge. Subsequent to standing for $5 \mathrm{~min}$ at $37^{\circ} \mathrm{C}$ to collect the cells at the tip of the EP tube, $\mathrm{T} 1$ and $\mathrm{T} 2$ sequence images were collected via $1.5 \mathrm{~T}$ nuclear magnetic resonance imaging (MRI) scans (Siemens AG, 
Munich, Germany). The data from the MRI scans were input into Gammaplan 5.3 (Leksell Perfexion Gamma Knife; Elekta Instrument AB, Stockholm, Sweden) to establish the most appropriate therapy for each patient. The cell-collecting site was wiped with $99 \%$ isodose, and 6-Gy peripheral doses were applied. Irradiation was applied with the Leksell Gamma Knife $^{\circledR}$ Perfexion ${ }^{\mathrm{TM}}$, and the cells were collected in $10-\mathrm{cm}$ culture dishes for continuous culture under sterile conditions subsequent to irradiation. For temozolomide treatment, the cells were treated with temozolomide at the $\mathrm{IC}_{50}$ dose $(5 \mathrm{mmol} / \mathrm{l})$ for $24 \mathrm{~h}$.

Protein extraction and western-blot analysis. The cells were harvested $24 \mathrm{~h}$ after treatment, and the total protein from the glioma cell line was extracted using radioimmunoprecipitation assay lysis buffer with proteinase inhibitor. The homogenates were clarified by centrifugation at $20,000 \times \mathrm{g}$ and $4^{\circ} \mathrm{C}$ for $15 \mathrm{~min}$, and the protein concentration was measured using the bicinchoninic acid method. A total of $40 \mathrm{mg}$ protein mixed with 5X SDS loading buffer was loaded into each lane and separated by 10 or $12 \%$ SDS-PAGE. The separated proteins were transferred to polyvinylidene fluoride membranes (Roche Diagnostics, Basel, Switzerland), Membranes were blocked with $5 \%$ non-fat milk in PBST $\left(1 \mathrm{~h}\right.$ at $\left.37^{\circ} \mathrm{C}\right)$, prior to incubation overnight at $4^{\circ} \mathrm{C}$ with primary antibodies against matrix metalloproteinase (MMP)-9 (1:1,000, cat . no. ab38898), and vascular endothelial growth factor (VEGF; 1:1,000, cat no. ab32152) (both from Abcam, Cambridge, UK) and EGFRvIII (rabbit monoclonal antibody, dilution 1:1,000; cat. no. sc-31156; Santa Cruz Biotechnology, Inc., Dallas, TX, USA) followed by incubation for $1 \mathrm{~h}$ at room temperature with a horseradish peroxidase-conjugated secondary antibody (cat. no. ZB2010, dilution 1:5,000; Zhongshan Bio Corp., Beijing, China). The membranes were washed in PBST. Subsequent to washing with stripping buffer, the membranes were re-probed with antibodies against GAPDH (dilution 1:5,000; cat. no. SC-47724; Santa Cruz Biotechnology, Inc.) at room temperature, overnight, and proteins were detected with an enhanced chemiluminescence detection system (Sigma-Aldrich; Merck KGaA, Darmstadt, Germany).

Intracranial animal model. In total, 3 groups of 4-week-old $(15 \mathrm{~g})$ female nude mice (Cancer Institute of the Chinese Academy of Medical Science). Each group contained 8 mice. Mice were kept at $2 /$ cage at a constant temperature $\left(24^{\circ} \mathrm{C}\right)$ with a 12 -h light-dark cycle (dark period from 08:00 a.m. to 20:00 p.m.). The mice were fed (specific pathogen free mice food) once a day $(20 \mathrm{~g})$ and were intracranially implanted with $5 \times 10^{5}$ U87-EGFRvIII cells to form the following groups: A group pre-treated with radiosurgery, a group treated with a combination therapy of 6 Gy radiosurgery and $\mathrm{IC}_{50}$ temozolomide, and a group with a negative control segment, using the Leksell Perfexion Gamma Knife. Bioluminescence imaging was used to detect intracranial tumour growth: Mice were anesthetized, injected intraperitoneally with D-luciferin (Promega Corporation) at $50 \mathrm{mg} / \mathrm{ml}$. Images were captured with the IVIS Imaging System (Caliper Life Sciences; PerkinElmer, Inc., Waltham, MA, USA) for 10-120 sec. To quantify the bioluminescence, identical circular regions of interest were drawn around the entire head of
Table I. Patient characteristics $(n=57)$.

\begin{tabular}{|c|c|}
\hline Characteristic & Value \\
\hline Median age at diagnosis (range), years & $49(22-76)$ \\
\hline \multicolumn{2}{|l|}{ Age groups by years, $\mathrm{n}(\%)$} \\
\hline $18-39$ & $37(65)$ \\
\hline $40-49$ & $10(18)$ \\
\hline $50-59$ & $3(5)$ \\
\hline $60-69$ & $4(7)$ \\
\hline$>70$ & $3(5)$ \\
\hline \multicolumn{2}{|l|}{ Sex } \\
\hline Male & $30(53)$ \\
\hline Female & $27(47)$ \\
\hline \multicolumn{2}{|l|}{ KPS score } \\
\hline Average & 73 \\
\hline Range & $20-90$ \\
\hline$\leq 70, \mathrm{n}(\%)$ & $20(35)$ \\
\hline$>70, \mathrm{n}(\%)$ & $37(65)$ \\
\hline \multicolumn{2}{|l|}{ Surgery } \\
\hline Total resection & $34(60)$ \\
\hline Partial resection & $23(40)$ \\
\hline \multicolumn{2}{|l|}{ Side } \\
\hline Left & $26(46)$ \\
\hline Right & $31(54)$ \\
\hline \multicolumn{2}{|l|}{ Survival, months } \\
\hline Average & 11.2 \\
\hline Range & 2-39 \\
\hline$<12, \mathrm{n}(\%)$ & $35(61)$ \\
\hline$\geq 12, \mathrm{n}(\%)$ & $22(39)$ \\
\hline \multicolumn{2}{|l|}{ WHO grade } \\
\hline I-II & $21(37)$ \\
\hline III & $22(39)$ \\
\hline IV & $14(25)$ \\
\hline
\end{tabular}

WHO, World Health Organization; KPS, Karnofsky performance status.

each animal, and the integrated flux of photons, photons per second, in each region of interest was determined using the Living Images software package 3.2 (Caliper Life Sciences; PerkinElmer, Inc.). Data were normalized to the bioluminescence at the initiation of treatment for each animal. A tumour volume of $0.6 \mathrm{~cm}^{3}$ and a weight of $20 \mathrm{~g}$ were set as the humane end-points. All protocols involving animals were performed in accordance with an approved Institutional Animal Care and Use Committee protocol (Cancer Institute of the Chinese Academy of Medical Science).

Immunohistochemistry analysis. Histological sections of tumour xenografts were excised and fixed in $10 \%$ neutral $\left(4^{\circ} \mathrm{C}\right)$ buffered formalin for 3 days. Following paraffin embedding, $5-\mu \mathrm{m}$ sections were cut. The paraffin-embedded tissue sections were used to examine EGFRvIII (cat. no. ab24293), EGFR (cat. no. ab52894), RAC- $\alpha$ serine/threonine-protein kinase 
Table II. Associations between clinic pathological features and EGFR and EGFRvIII status $(\mathrm{n}=57)$.

\begin{tabular}{|c|c|c|c|c|}
\hline Characteristic & EGFR-positive, n (\%) & P-value & EGFRvIII-positive, n (\%) & P-value \\
\hline Patients & $42(74)$ & & $29(51)$ & \\
\hline \multicolumn{5}{|l|}{ Age, years } \\
\hline $18-49$ & $26(46)$ & 0.426 & $20(35)$ & 0.514 \\
\hline $50-76$ & $16(28)$ & & $9(16)$ & \\
\hline \multicolumn{5}{|l|}{ Sex } \\
\hline Male & $23(40)$ & 0.590 & $16(28)$ & 0.696 \\
\hline Female & $19(33)$ & & $13(23)$ & \\
\hline \multicolumn{5}{|l|}{ KPS score } \\
\hline$\leq 70$ & $16(28)$ & 0.426 & $8(14)$ & 0.227 \\
\hline$>70$ & $26(46)$ & & $21(37)$ & \\
\hline \multicolumn{5}{|l|}{ Surgery } \\
\hline Total resection & $29(51)$ & $0.016^{\mathrm{a}}$ & $17(30)$ & 0.872 \\
\hline Partial resection & $13(23)$ & & $12(21)$ & \\
\hline \multicolumn{5}{|l|}{ Side } \\
\hline Left & $19(33)$ & 0.924 & $16(28)$ & 0.140 \\
\hline Right & $23(40)$ & & $13(23)$ & \\
\hline \multicolumn{5}{|l|}{ Survival, months } \\
\hline$<12$ & $30(53)$ & $0.009^{\mathrm{b}}$ & $22(39)$ & $0.022^{\mathrm{a}}$ \\
\hline$\geq 12$ & $12(21)$ & & $7(12)$ & \\
\hline \multicolumn{5}{|l|}{ WHO grade } \\
\hline $\mathrm{I}-\mathrm{II}$ & $12(21)$ & $0.030^{\mathrm{a}}$ & $7(12)$ & $0.043^{\mathrm{a}}$ \\
\hline III-IV & $30(53)$ & & 22 (39) & \\
\hline
\end{tabular}

${ }^{\mathrm{a}} \mathrm{P}<0.05 ;{ }^{\mathrm{b}} \mathrm{P}<0.01$. EGFR, epidermal growth factor receptor; EGFRvIII, EGFR variant III; WHO, World Health Organization; KPS, Karnofsky performance status.

(AKT-1; cat. no. ab81283), MMP-2 (cat. no. ab37150), MMP-9 (cat. no. ab38898) and X-ray repair cross-complementing protein 6 (Ku70; cat. no. ab92450) expression. Subsequent to de-waxing, sections were incubated with primary antibodies (all diluted to 1:100; and all provided by Abcam) overnight at $4^{\circ} \mathrm{C}$, followed by incubation with abiotin-labelled secondary antibody (dilution 1:100; cat. no. SPN-9001; Zhongshan Bio Corp., Beijing, China) for $1 \mathrm{~h}$ at $37^{\circ} \mathrm{C}$, incubation with diaminobenzidine for $2 \mathrm{~h}$ at room temperature, counterstaining with haematoxylin for $10 \mathrm{~min}$ at room temperature, dehydration and visualization using light microscopy (Olympus Corporation, Tokyo, Japan).

Statistical analysis. SPSS software (v.16.0; SPSS Inc., Chicago, IL, USA) was used for all statistical analyses. One-way analysis of variance with a Tukey's post hoc test or was used to evaluate the differences between groups. $\mathrm{P}<0.05$ was considered to indicate a statistically significant difference.

\section{Results}

Statistical results from clinical data. The results (Table II) indicate that $26(46 \%)$ patients were younger than the median age of 49 years, and $16(28 \%)$ were older $(\mathrm{P}=0.426)$. A total of 23 patients were men $(40 \%)$ and 19 were women $(33 \% ; \mathrm{P}=0.590)$. The number of patients with a KPS score lower than the mean score of 70 was $16(28 \%)$, and the number of patients with a KPS score higher than the mean score was $26(46 \% ; \mathrm{P}=0.426)$. A total of 29 patients (51\%), underwent complete tumour resection, and 13 (23\%) patients underwent partial tumor resection $(\mathrm{P}=0.016)$. There were 19 patients with tumours growing on the left cerebral hemisphere (33\%) and 23 patients with tumours growing on the right cerebral hemisphere $(40 \% ; \mathrm{P}=0.924)$. The number of patients with a survival time of $<12$ months, the median survival time was $30(53 \%)$, and the number of patients with a survival time $>12$ months was $12(21 \%$; $\mathrm{P}=0.009)$. The number of patients with WHO grades I-II was 12 (21\%) and the number of patients with WHO grades III-IV was 30 (53\%; $\mathrm{P}=0.030$ ). Statistical analysis was performed on the data showing the levels of expression of EGFR and EGFRvIII from the pathology results of the tumours of 57 patients (Table II). From the 57 patients, $29(51 \%)$ exhibited high expression of EGFRvIII. Of these patients, 20 and 9 were younger and older than the median age, respectively. In this group, 16 were men (28\%), and 13 were women $(23 \% ; \mathrm{P}=0.696)$. The number of the patients with a KPS score $<70$, the mean score, was $8(14 \%)$, and the number of patients with a KPS score $>70$ was $21(37 \% ; \mathrm{P}=0.227)$. A total of 17 patients $(30 \%)$ underwent complete tumour resection and $12(21 \%)$ patients underwent a partial tumour resection $(\mathrm{P}=0.872)$. A total of 16 patients exhibited tumours growing on the left cerebral hemisphere (28\%) and 13 patients exhibited tumours growing on the right 


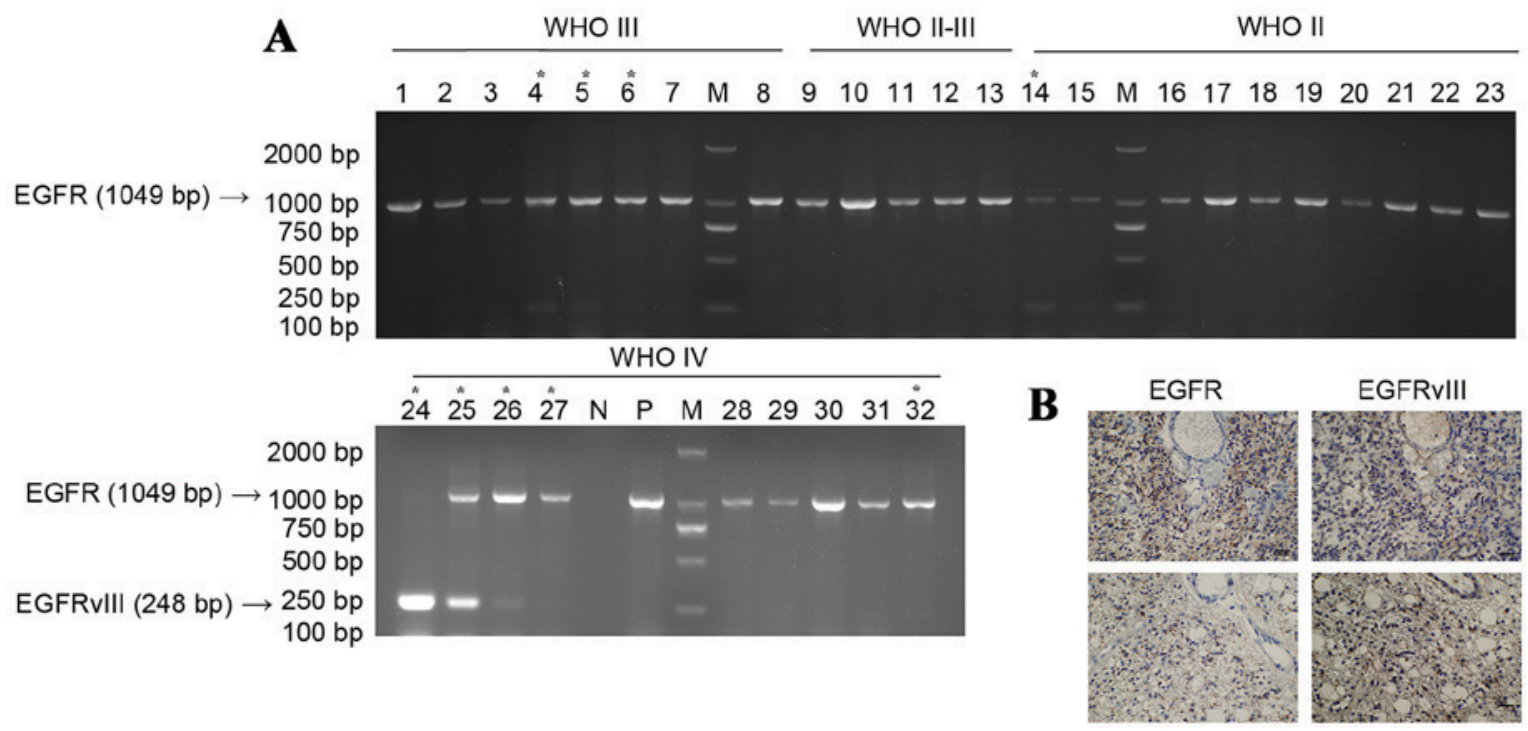

Figure 1. (A) Agarose gel electrophoresis of tumour polymerase chain reaction products. "Indicates the tumours expressing EGFRvIII. (B) Post-operative histology of EGFR and EGFRvIII tumours. EGFR, epidermal growth factor receptor; EGFRvIII, EGFR variant III mutation; $\mathrm{N}$, dd $\mathrm{H}_{2} \mathrm{O}$ negative control; P, EGFRvIII amplification product control; M, marker.

cerebral hemisphere (23\%; $\mathrm{P}=0.140)$. The number of patients with a survival time less than the median survival time was $22(39 \%)$ and the number of patients with a survival time greater than the median survival time was $7(12 \% ; \mathrm{P}=0.022)$. The number of patients with WHO grades I-II was $7(12 \%)$ and the number of patients with WHO grades III-IV was 22 (39\%; $\mathrm{P}=0.043)$.

It was revealed that the expression of EGFR and EGFRvIII demonstrated no significant correlation with the patient age, gender, KPS score, and growth position. There was a significant correlation observed between range of surgical resection and high expression levels of EGFR, EGFRvIII, patient survival time and tumour grade. It was evident that the high expression of EGFR and EGFRvIII was negatively correlated with patient survival time and mostly occurred in high-grade glioma $(\mathrm{P}<0.05$ and $\mathrm{P}<0.01$, respectively).

Agarose gel electrophoresis of tumour specimen PCR products. As indicated by the results from agarose gel electrophoresis (Fig. 1), among the 32 patients with tumours, 9 patients (28\%) experienced relatively marked expression of EGFRvIII, 1 (3\%) of whom possessed a WHO grade II tumour and $8(25 \%)$ of whom possessed a WHO grade III-IV tumour, which was not completely consistent with the data from the clinical pathology results. The reason for this observation maybe the position from which the tumour specimens were obtained. However, it was observed that the high expression of EGFRvIII was most common in high-grade glioma, indicating that high expression of EGFRvIII demonstrates a correlation with glioma malignancy.

Expression of EGFRvIII, EGFR, AKT-1, MMP-2, MMP-9 and $K u 70$ in in vitro experiments. In addition to EGFRvIII as the typical marker for the cell invasion index, MMP may also be detected. Ku70 is a eukaryotic protein involved in the repair of DNA double-strand breaks by non-homologous end-joining (12), and maybe a critical biomarker for cancer
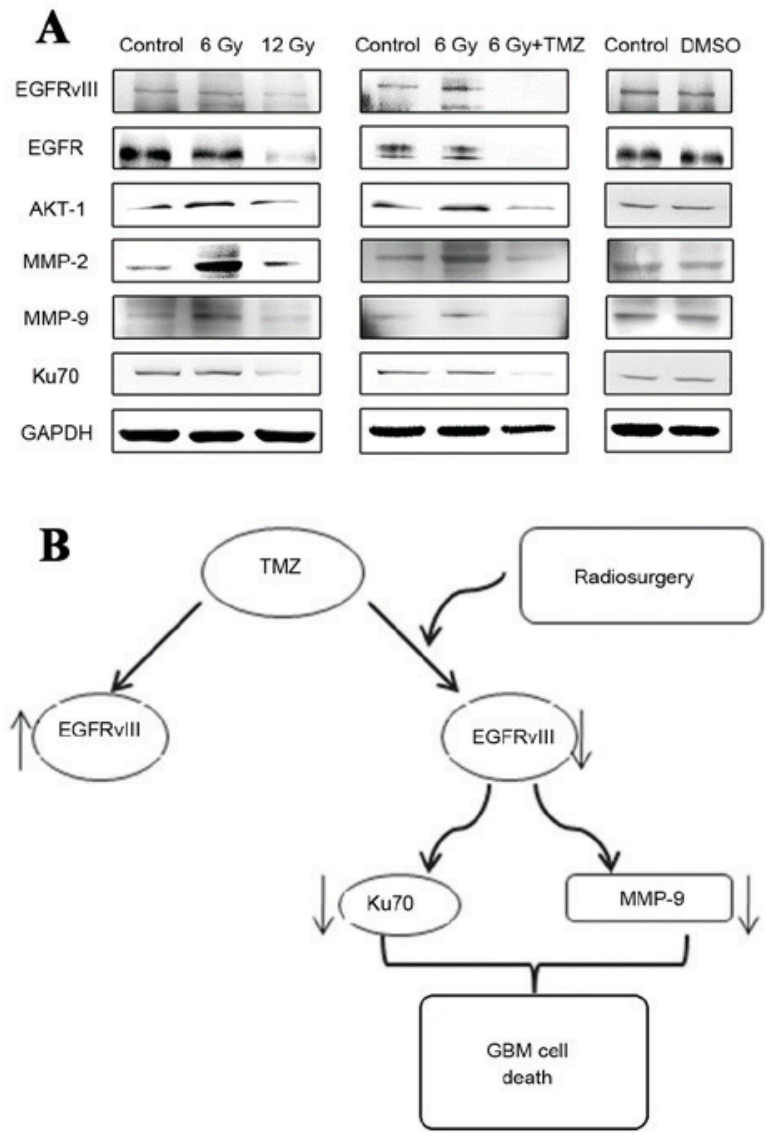

Figure 2. In vitro expression of EGFRvIII, EGFR, AKT-1, MMP-2, MMP-9 and Ku70. (A) Levels of expression of EGFRvIII, EGFR, AKT-1, MMP-2, MMP-9 and Ku70 proteins in vitro in U87-EGFRvIII cell lines treated with negative control, 6 Gy radiation, 12 Gy radiation, and combined treatment of 6 Gy radiosurgery and temozolomide. (B) Schematic diagram illustrating the combined treatment of radiosurgery and temozolomide. EGFR, epidermal growth factor receptor; EGFRvIII, EGFR variant III; AKT-1, RAC- $\alpha$ serine/threonine-protein kinase; MMP-2, matrix metalloproteinase 2;MMP-9; $\mathrm{Ku} 70, \mathrm{X}$-ray repair cross-complementing protein 6; GMB, glioblastoma multiforme; DMSO, dimethyl sulphoxide; TMZ, temozolomide. 
$\mathbf{A}$

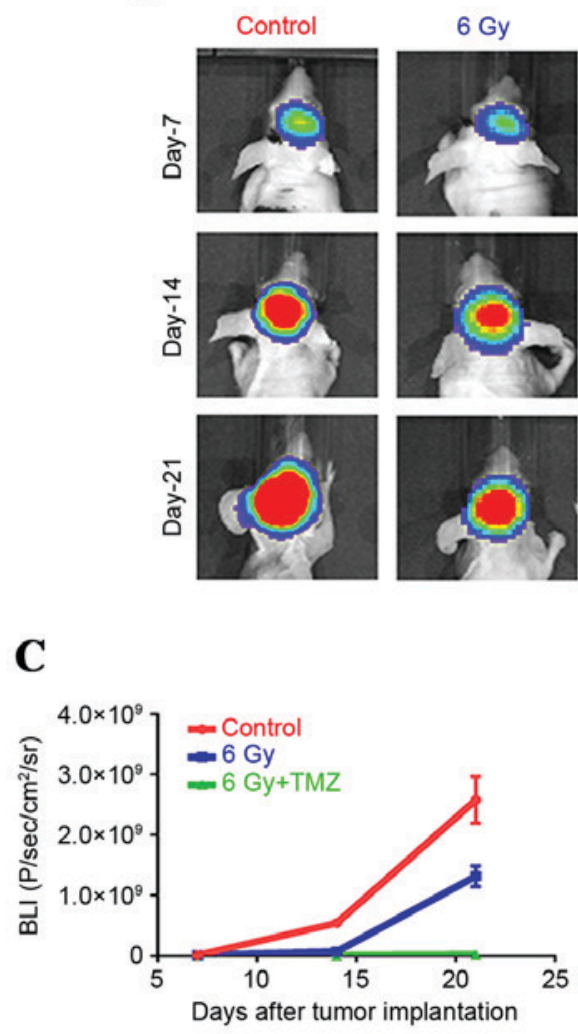

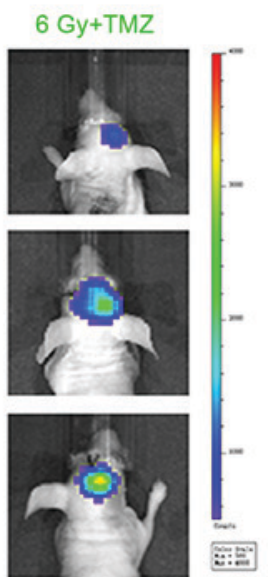

B

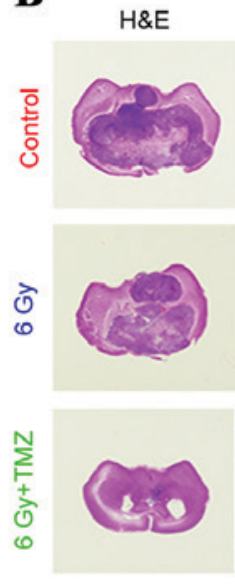

EGFRvIII

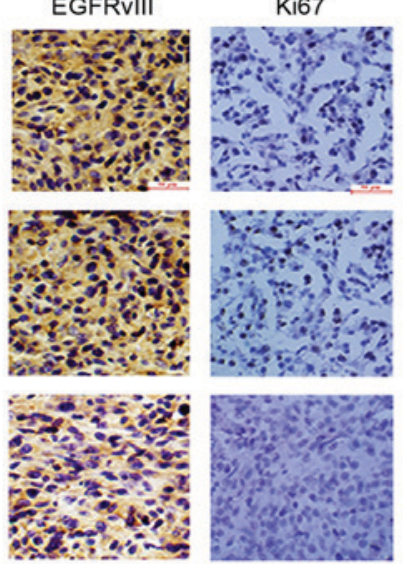

C
D

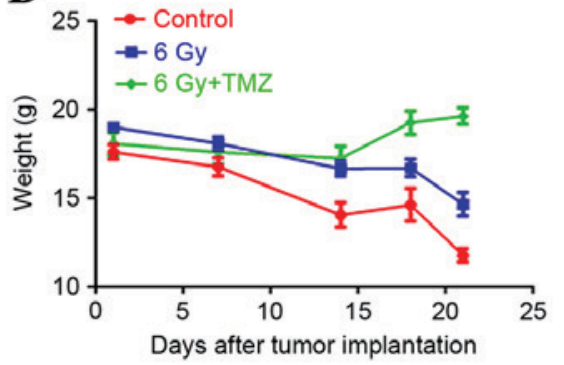

$\mathbf{E}$

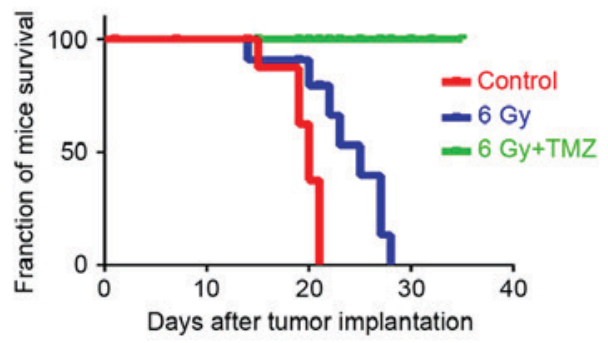

Figure 3. U87-EGFRvIII cell line in heterotransplantation models treated with negative control, 6 Gy radiation, and combined treatment with radiosurgery and temozolomide. (A) Bioluminescence images from heterotransplantation models (magnification, $\mathrm{x} 40$ ) treated with negative control, 6 Gy radiation (48 h after treatment with 6 Gy peripheral doses by radiosurgery with $99 \%$ of isodose) and combined treatment with radiosurgery and temozolomide (cells were treated for $24 \mathrm{~h}$ with temozolomide at the $\mathrm{IC}_{50}$ dose for $24 \mathrm{~h}$ after $6 \mathrm{~Gy}$ radiation) at 7, 14 and 21 days subsequent to tumour development. (B) Histological sections of tumor xenografts were excised and fixed in $10 \%$ neutral buffered formalin. Following paraffin embedding, 5- $\mu$ m sections were cut and dried, deparaffinized, and rehydrated. Next, the nonspecific binding sites were blocked, and the slides were incubated at $4^{\circ} \mathrm{C}$ overnight in a $1: 100$ dilution of primary antibodies. Finally, the slides were incubated with a secondary antibody for $30 \mathrm{~min}$. Immunohistochemistry for EGFRvIII and Ki-67 on xenograft tumour sections (Scale bar, $100 \mu \mathrm{m}$ ). (C) Evaluation of tumour growth curves. (D) Evaluation of weights of mice. (E) Improved survival observed in mice treated with the combined therapy. H\&E, hematoxylin and eosin; TMZ, temozolomid; EGFRvIII, epidermal growth factor receptor variant III.

cell sensitivity to DNA damage response. The results of western blot analysis (Fig. 2) indicated that under radiation treatment at a low dose of $6 \mathrm{~Gy}$, the expression levels of the EGFRvIII, AKT-1, MMP-2 and MMP-9 proteins in U87-EGFRvIII cell lines were upregulated to varying extents, with the exception of EGFR and Ku70. The results from these two markers requires additional study, which is generally consistent with the results reported in the literature that radiation treatment at low doses may lead to an increase in glioma cell invasiveness (13). However, subsequent to doubling of the radiation dose, the expression levels of the proteins demonstrated marked downregulation. Subsequent to radiosurgery in combination with temozolomide treatment at the $\mathrm{IC}_{50}$ dose, the expression levels of the EGFRvIII, EGFR, AKT-1, MMP-2, MMP-9 and Ku70 proteins in the EGFRvIII cell lines exhibited evident downregulation, and the combined therapy was able to reverse the condition in which the low dose of 6 Gy promoted the upregulated expression of the proteins. In the combined therapy group, the expression of EGFRvIII, EGFR, AKT-1, MMP-2, MMP-9 and $\mathrm{Ku} 70$ in the EGFRvIII cell lines was significantly lower compared with the simple radiosurgery or simple temozolomide treatment groups.
U87-EGFRvIII cells in heterotransplantation models. In the heterotransplantation animal models, it was observed that in the U87-EGFRvIII cells treated by radiotherapy at the low dose of $6 \mathrm{~Gy}$, the tumorigenicity was weaker compared with the cells treated with the negative control, which differed from the results of the in vitro experiments. Although radiation treatment at the low dose may promote the upregulation of expression of certain invasiveness indices of U87-EGFRvIII cells such as the AKT-1 and MMP-2, while it exhibits certain inhibitory effects on tumour proliferation. The mechanism is unclear, but in animal experiments in the present study, different doses of radiation therapy have a significant inhibitory effect on the growth of the tumour (Fig. 3). And, the size of the tumours formed in the U87-EGFRvIII cells treated with the combination of radiosurgery and temozolomide were smaller compared with those in the cells treated with 6 Gy radiation. No significant change was observed in the weight of the nude mice in the combined treatment group, and their survival rate was higher compared with the nude mice with U87-EGFRvIII cells treated with the 6 Gy radiation treatment. The results from the immunohistochemical analysis of the tumour cells (Fig. 3) in the heterotransplantation models indicated that the expression of EGFRvIII and Ki-67 in the tumour 
tissues subsequent to tumour formation in the U87-EGFRvIII cells treated with a combination of radiosurgery and temozolomide demonstrated a marked downregulation, and subsequent to combined treatment, the inhibition of MMP-9, VEGF and EGFRvIII was more evident. These results are different compared with those from the in vitro experiments, indicating that subsequent to the treatment of the U87-EGFRvIII cells, the proliferation and tumour formation abilities decreased significantly, and the invasion abilities of the formed tumours were also inhibited.

In the present study, the EGFRvIII mutation mostly occurred in GBMs with higher malignancies, and the combined treatment of radiosurgery and temozolomide may inhibit the invasiveness of tumour cells compared with other treatment regimens subsequent to tumour formation in the U87-EGFRvIII cells. The combined radiosurgery and temozolomide therapy also exerts a stronger inhibitory effect on the tumorigenicity of the U87-EGFRvIII cells in the brains of nude mice.

\section{Discussion}

GBM is the one of most invasive types of tumours in the human brain. The invasion capabilities of these tumour cells results in difficulties in achieving a complete surgical resection of the tumour, and high rates of recurrence and mortality. Therefore, patients with GMB exhibit poor prognoses and notably short median survival times. The EGFR-targeting tyrosine kinase inhibitor possesses a high degree of immune activity, which is usually associated with drug resistance and may lead to chemotherapy failure. EGFR is the tyrosine kinase receptor on the cell surface and exhibits significantly high expression levels and mutations in GBM. The most common type of EGFR mutation is EGFRvIII: The deletion of exon 2 to 7, which leads to the fusion of exon 1 and 8 and produces tumour-specific surface antigens on the cell surface $(14,15)$. EGFRvIII has also been identified in other types of cancer, including lung, breast and prostate cancer, and may also be present in head and neck squamous carcinoma, but it is not exhibited in normal tissues or benign tumour tissues (14-16). The amplification of EGFR is often accompanied by the upregulated expression of EGFRvIII, which is significantly correlated with short survival times for patients with BMG. Previous studies have indicated that a high expression level of EGFRvIII is positively correlated with the invasion abilities of GBM cells. Previous studies have also indicated that $30-60 \%$ of GBM patients exhibit high expression levels of EGFRvIII, and these mutations are not detected in normal brain tissues of adult humans or other normal tissues (17-19). Therefore, EGFRvIII is a good target for GBM gene therapy. However, limited studies are available on the molecular biology of EGFRvIII-associated mechanisms (20-22). Numerous previous studies have examined the mechanisms and development of EGFRvIII-associated target drugs, such as the use of the protein Jagged 1-Notch signalling pathway and antibodies that specifically identify EGFR DNA oligonucleotide aptamers, among others, for the development of a more effective therapeutic regimen due to the poor effectiveness of current therapeutic tools $(21,22)$. At present, surgical resection combined with chemotherapy remains the best treatment, although it is difficult to implement the enhanced radiation treatment combined with temozolomide to improve the therapeutic effect $(23,24)$. In the present study, data from the clinical cases in the combined treatment group indicated that in the low-grade, WHO I-II, gliomas, 33\% of the tumours possessed the EGFRvIII mutation. In the high-grade, WHO III-IV, gliomas, $61 \%$ of the tumours exhibited the EGFRvIII mutation. It was revealed that the probability of occurrence of high-grade glioma with the EGFRvIII mutation was greater, indicating that the EGFRvIII mutation was associated with tumour malignancy. PCR analysis of the tumour cells indicated that 9 patients $(28 \%)$ exhibited EGFRvIII expression in tumour tissues, $1(11 \%)$ of which possessed a WHO grade I-II tumour and 8 (89\%) of which possessed WHO grade III-IV tumours. This proportion was lower compared with the results from pathological tests, which may be due to uncertainty in the sampling positions, but this method also illustrated the correlation between EGFRvIII mutation and tumour malignancy.

In the glioma radiation treatment group, the observation that therapeutic efficacy is positively correlated with radiation dose was verified. In the present study, the analysis of high-dose radiotherapy was not performed as the results are well established. In addition, under the conditions that normal brain tissues are protected and the radiation reactions are reduced, little improvement remains to be achieved in current radiotherapy measures $(25,26)$. Certain previous studies have indicated that radiation treatment at low doses between 5-8 Gy exhibits a promotional effect on the invasion abilities of tumour cells. Radiation treatment may promote the invasion abilities of glioma cells $(13,27,28)$. Therefore, 6 Gy was selected as the dose for the present study to increase the invasion abilities of glioma. The results of the western blot analysis indicated that the 6-Gy dose may increase the expression levels of the EGFRvIII, EGFR, AKT-1, MMP-2, MMP-9 and $\mathrm{Ku} 70$ proteins in the U87-EGFRvIII cell lines, but subsequent to combined therapy with temozolomide, the expression levels of these proteins in the U87-EGFRvIII cell lines was significantly inhibited and was evidently lower compared with the normal negative control group. The animal experiments illustrated the marked difference. The 6-Gy radiation dose of the in vitro experiment increased the invasion capabilities of the U87-EGFRvIII cell lines, but the tumorigenicity and the invasive indices in the tumour tissues subsequent to tumour formation were not significantly improved and were inhibited, indicating that low-dose radiation, although increasing the invasiveness of the U87-EGFRvIII cells, did not significantly increase the rates of proliferation and the growth of the U87-EGFRvIII cells, but demonstrated an inhibitory effect. Subsequent to combined radiotherapy and temozolomide treatment, the tumour growth in the brains of the nude mice was significantly lower and the survival time was greatly extended compared with those in the negative control treatment group and the radiation-only treatment group. These results suggested that combined treatment with radiotherapy and temozolomide exhibits a clear inhibitory effect on the tumourigenicity of U87-EGFRvIII cells.

Overall, gene-targeted therapy based on molecular biology and epigenetics is a potential prospect for GBM treatment, but the application of this approach in clinical treatment is not available at present. Therefore, to improve the existing treatment options and to enhance the efficacy of current 
therapeutics, the development of more accurate radiotherapy treatments or the use of additional chemotherapeutical drugs, including bevacizumab and valproic acid (VPA) (29-31), should be investigated alongside the development of novel therapeutic techniques and treatments.

\section{Acknowledgements}

The temozolomide was donated by Tasly Pharmaceutical Co., Ltd. (Tianjin, China).

\section{Funding}

The present study was supported by the National High-tech Research and Development Program of China (863 Program; grant no. 2014AA021102), the General Item of the Tianjin Natural Science Fund (grant no. 13JCYBJC24000), the Science and Technology Research and Development Program of Hebei Province of China (grant no. 13277785D), and the Science and Technology Fund of the Tianjin Health Bureau (grant no. 2011KZ95).

\section{Availability of data and materials}

All data generated or analyzed during this study are included in this published article.

\section{Authors' contributions}

All authors read and approved the final manuscript. Cell lines and tissue samples:JLX, YLT, GKW and CF. Cell treatment :YGL,GKW, ZYZ, YLG, YQZ, DSX and XML. Immunohistochemistry analysis: YGL, KZ, XML and CSK. Intracranial animal model: YGL, JHZ, KZ and CSK. Protein extraction and western-blot analysis: YGL and KZ. RT-PCR: YGL, JHZ and KZ. Statistical analysis: YGL, JLX, JHZ and KZ. Writing the manuscript: YGL, JHZ, JLX and XML.

\section{Ethics approval and consent to participate}

The present study was approved by the Ethics Committee of the Second Hospital of Tianjin Medical University (Tianjin, China). Approval for the use of glioma samples was obtained from each patient.

\section{Consent for publication}

Not applicable.

\section{Competing interests}

The authors declare that they have no competing interests.

\section{References}

1. Furnari FB, Fenton T, Bachoo RM, Mukasa A, Stommel JM, Stegh A, Hahn WC, Ligon KL, Louis DN, Brennan C, et al: Malignant astrocytic glioma: Genetics biology, and paths to treatment. Genes Dev 21: 2683-2710, 2007.

2. Maher EA, Furnari FB, Bachoo RM, Rowitch DH, Louis DN Cavenee WK and DePinho RA: Malignant glioma: Genetics and biology of a grave matter. Genes Dev 15: 1311-1333, 2001.
3. Wen PY and Kesari S: Malignant gliomas in adults. N Engl J Med 359: 492-507, 2008.

4. Jin X, Yin J, Kim SH, Sohn YW, Beck S, Lim YC, Nam DH, Choi YJ and Kim H: EGFR-AKT Smad signaling promotes formation of glioma stem-like cells and tumor angiogenesis by ID3-driven cytokine induction. Cancer Res 71: 7125-7134, 2011.

5. Jeon HM, Kim SH, Jin X, Park JB, Kim SH, Joshi K, Nakano I and Kim H: Cross talk between glioma-initiating cells and endothelial cells drives tumor progression. Cancer Res 74: 4482-4492, 2014.

6. Moscatello DK, Holgado-Madruga M, Godwin AK, Ramirez G, Gunn G, Zoltick PW, Biegel JA, Hayes RL and Wong AJ: Frequent expression of a mutant epidermal growth factor receptor in multiple human tumors. Cancer Res 55: 5536-5539, 1995.

7. Libermann TA, Nusbaum HR, Razon N, Kris R, Lax I, Soreq H, Whittle N, Waterfield MD, Ullrich A and Schlessinger J: Amplification, enhanced expression and possible rearrangement of EGF receptor gene in primary human brain tumours of glial origin. Nature 313: 144-147, 1985.

8. Hatanpaa KJ, Burma S,Zhao D and Habib AA: Epidermal growth factor receptor in glioma: Signal transduction, neuropathology, imaging, and radioresistance. Neoplasia 12: 675-684, 2010.

9. Pelloski CE, Ballman KV, Furth AF, Zhang L, Lin E, Sulman EP, Bhat K, McDonald JM, Yung WK, Colman H, et al: Epidermal growth factor receptor variant III status defines clinically distinct subtypes of glioblastoma. J Clin Oncol 25: 2288-2294, 2007.

10. Yoshimoto K, Dang J, Zhu S, Nathanson D, Huang T, Dumont R, Seligson DB, Yong WH, Xiong Z, Rao N, et al: Development of a real-time RT-PCR assay for detecting EGFRvIII in glioblastoma samples. Clin Cancer Res 14: 488-493, 2008.

11. Louis DN, Perry A, Reifenberger G, von Deimling A, Figarella-Branger D, Cavenee WK, Ohgaki H, Wiestler OD, Kleihues P and Ellison DW: The 2016 World Health Organization classification of tumors of the central nervous system: A summary. Acta Neuropathol 131: 803-820, 2016.

12. Jia Q, Li Y, Xu D, Li Z, Zhang Z, Zhang Y, Liu D, Liu X, Pu P and Kang C: Radiosensitivity of glioma to Gamma Knife treatment enhanced in vitro and in vivo by RNA interfering Ku70 that is mediated by a recombinant adenovirus. J Neurosurg 113 (Suppl): S228-S235, 2010.

13. Madani I, De Neve W and Mareel M: Does ionizing radiation stimulate cancer invasion and metastasis? Bull Cancer 95: 292-300, 2008.

14. GeH,Gong X and Tang CK: Evidence of high incidence of EGFRvIII expression and coexpression with EGFR in human invasive breast cancer by laser capture microdissection and immunohistochemical analysis. Int J Cancer 98: 357-361, 2002.

15. Olapade-Olaopa EO, Moscatello DK, MacKay EH, Horsburgh T, Sandhu DP, Terry TR, Wong AJ and Habib FK: Evidence for the differential expression of a variant EGF receptor protein in human prostate cancer. Br J Cancer 82: 186-194, 2000.

16. Khattri A, Zuo Z, Brägelmann J, Keck MK, El Dinali M, Brown CD, Stricker T, Munagala A, Cohen EE, Lingen MW, et al: Rare occurrence of EGFRvIII deletion in head and neck squamous cell carcinoma. Oral Oncol 51: 53-58, 2015.

17. Tan Y, Shi YS, Wu XD, Liang HY, Gao YB, Li SJ, Zhang XM, Wang F and Gao TM: DNA aptamers that target human glioblastomamultiforme cells overexpressing epidermal growth factor receptor variant III in vitro. Acta Pharmacol Sin 34: 1491-1498, 2013.

18. Kim J, Lee JS, Jung J, Lim I, Lee JY and Park MJ: Emodin suppresses maintenance of stemness by augmenting proteosomal degradation of epidermal growth factor receptorepidermal growth factor receptor variant III in glioma stem cells. Stem Cells Dev 24: 284-295, 2015.

19. Congdon KL, Gedeon PC, Suryadevara CM, Caruso HG, Cooper LJ, Heimberger AB and Sampson JH: Epidermal growth factor receptor and variant III targeted immunotherapy. Neuro Oncol 16 (Suppl 8): viii20-viii25, 2014.

20. Zhang X, Liang H, Tan Y, Wu X, Li S and Shi Y: U87-EGFRvIII cell-specific aptamer mediates small interfering RNA delivery. Biomed Rep 2: 495-499, 2014.

21. Kim EJ, Kim SO, Jin X, Ham SW, Kim J, Park JB, Lee JY, Kim SC and Kim H: Epidermal growth factor receptor variant III renders glioma cancer cells less differentiated by JAGGED1. Tumour Biol 36: 2921-2928, 2015.

22. Katanasaka Y, Kodera Y, Kitamura Y, Morimoto T, Tamura T and Koizumi F: Epidermal growth factor receptor variant type III markedly accelerates angiogenesis and tumor growth via inducing c-myc mediated angiopoietin-like 4 expression in malignant glioma. Mol Cancer 12: 31, 2013. 
23. Senft C, Bink A, Franz K, Vatter H, Gasser T and Seifert V: Intraoperative MRI guidance and extent of resection in glioma surgery: A randomised, controlled trial. Lancet Oncol 12: 997-1003, 2011.

24. Gilbert MR, Dignam JJ, Armstrong TS, Wefel JS, Blumenthal DT, Vogelbaum MA, Colman H, Chakravarti A, Pugh S, Won M, et al: A randomized trial of bevacizumab for newly diagnosed glioblastoma. N Engl J Med 370: 699-708, 2014.

25. Binello E, Green S and Germano IM: Radiosurgery for high-grade glioma. Surg Neurol Int 3 (Suppl 2): S118-S126, 2012.

26. Waters JD, Rose B, Gonda DD, Scanderbeg DJ, Russell M, Alksne JF, Murphy K, Carter BS, Lawson J and Chen CC: Immediate post-operative brachytherapy prior to irradiation and temozolomide for newly diagnosed glioblastoma. J Neurooncol 113: 467-477, 2013.

27. Kwak SY, Kim BY, Ahn HJ, Yoo JO, Kim J, Bae IH and Han YH: Ionizing radiation-inducible miR-30e promotes glioma cell invasion through EGFR stabilization by directly targeting CBL-B. FEBS J 282: 1512-1525, 2015.

28. Zhang X, Li X, Zhang N, Yang Q and Moran MS: Low doses ionizing radiation enhances the invasiveness of breast cancer cells by inducing epithelial-mesenchymal transition. Biochem Biophys Res Commun 412: 188-192, 2011.
29. Iuchi T, Hatano K, Uchino Y, Itami M, Hasegawa Y, Kawasaki K, Sakaida T and Hara R: Methionine Uptake and required radiation dose to control glioblastoma. Int J Radiat Oncol Biol Phys 93: 133-140, 2015.

30. Hawkins-Daarud A, Rockne R, Corwin D, Anderson AR, Kinahan $\mathrm{P}$ and Swanson KR: In silico analysis suggests differential response to bevacizumab and radiation combination therapy in newly diagnosed glioblastoma. J R Soc Interface 12: 20150388, 2015

31. Krauze AV, Myrehaug SD, Chang MG, Holdford DJ, Smith S, Shih J, Tofilon PJ, Fine HA and Camphausen K: A phase 2 study of concurrent radiation therapy, temozolomide, and the histone deacetylase inhibitor valproic acid for patients with glioblastoma. Int J Radiat Oncol Biol Phys 92: 986-992, 2015.

cc) (i) (5) This work is licensed under a Creative Commons 